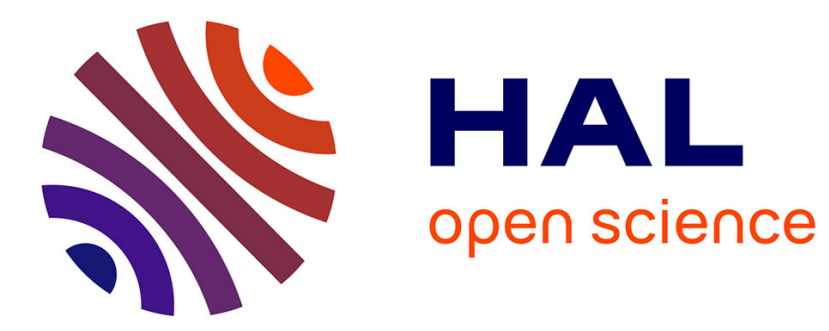

\title{
Musique et société en Asie du Sud
}

Christine Guillebaud

\section{To cite this version:}

Christine Guillebaud. Musique et société en Asie du Sud. L'Homme - Revue française d'anthropologie, 2004, Musique et Anthropologie, 171-172, pp.499 - 511. 10.4000/lhomme.24994 . halshs-01623506

\section{HAL Id: halshs-01623506 https://shs.hal.science/halshs-01623506}

Submitted on 25 Oct 2017

HAL is a multi-disciplinary open access archive for the deposit and dissemination of scientific research documents, whether they are published or not. The documents may come from teaching and research institutions in France or abroad, or from public or private research centers.
L'archive ouverte pluridisciplinaire HAL, est destinée au dépôt et à la diffusion de documents scientifiques de niveau recherche, publiés ou non, émanant des établissements d'enseignement et de recherche français ou étrangers, des laboratoires publics ou privés. 


\title{
L'HOMME L'Homme \\ Revue française d'anthropologie \\ 171-172 | 2004 \\ Musique et anthropologie
}

\section{Musique et société en Asie du Sud}

\author{
Christine Guillebaud
}

revues.org

Édition électronique

URL : http:///homme.revues.org/24994

ISSN : 1953-8103

\section{Éditeur}

Éditions de l'EHESS

\section{Édition imprimée}

Date de publication : 1 décembre 2004

Pagination : 499-511

ISSN : 0439-4216

Référence électronique

Christine Guillebaud, "Musique et société en Asie du Sud », L'Homme [En ligne], 171-172 | 2004, mis en ligne le 01 janvier 2006, consulté le 06 janvier 2017. URL : http:///homme.revues.org/24994 ; DOI :

10.4000//homme.24994

Ce document est un fac-similé de l'édition imprimée.

(c) École des hautes études en sciences sociales 


\title{
Musique et société en Asie du Sud
}

\author{
Christine Guillebaud
}

$E$

N 1964, Alan P. Merriam définissait l'ethnomusicologie comme l'étude de la musique dans la culture et en tant que culture (" music in and as culture ") ${ }^{1}$. Or, en Asie du Sud, rares sont les auteurs qui ont pensé l'objet musical en ces termes. Les pages qui suivent font l'état des lieux des travaux les plus importants des quinze dernières années. J'aborderai successivement la musicologie historique, les études portant sur les traditions classiques contemporaines, l'ethnomusicologie naissante des objets " non classiques ", les modèles performatifs et enfin les enjeux soulevés par l'étude des musiques dites «populaires» (popular music).

\section{La musicologie historique: le texte comme terrain d'ethnomusicologie?}

Les nombreuses sources écrites disponibles sur le terrain indien (inscriptions, ouvrages littéraires, traités et iconographie) renseignent tout au long de l'histoire pré-islamique et moghole sur la façon dont les lettrés formalisèrent à leur époque la théorie musicale ainsi que ses exégèses. Ces sources historiques, devenues objets privilégiés de la recherche musicale, ouvrent peu de brèches à une éventuelle approche "merriamiste" de la musique : parce que ces musiques appartiennent au passé, elles échappent à toute approche sensible de leur structure interne, à moins que les musicologues ne se réferent, pour pallier ce manque, aux pratiques contemporaines hindoustanie et carnatique de la musique classique. Même dans ce cas de figure, la réflexion musicologique a porté toute son attention sur la question de la dialectique entre théorie et pratique, beaucoup plus que sur celle du lien entre musique et société.

1. Alan P. Merriam, The Anthropology of Music, Evanston, Northwestern University Press, 1964. 
Ashok D. Ranade (2000) a récemment dégagé les caractéristiques générales de la musicologie historique (Music-history), encore appelée "modèle scolastique " ou "shastrique », du nom sanskrit désignant un traité. L'auteur la définit comme l'étude des "aspects non performatifs de la musique ", tout en soulignant à juste titre qu'elle nécessite cependant « de se tourner vers les cultures où la musique se pratique " (Ranade 2000 : 6). Son étude historique de la musique hindoustanie a nécessité une approche globale et chronologique des différentes sources écrites, ainsi que de l'histoire politique et culturelle de la période moghole (XVI ${ }^{\mathrm{e}}-\mathrm{XIX}^{\mathrm{e}}$ siècles). Ashok D. Ranade propose ainsi de recontextualiser ces sources par un examen de l'organisation politique, des mouvements de bhakti naissants et de l'idéologie soufie de l'époque.

Lewis E. Rowell (1992) présente son travail sur les sources pré-islamiques (voir aussi Widdess 1995) comme une " archéologie musicale » où l'étude des " faits de musique " (mélodies, rythmes, formes) encore appelés « organisation musicale » tiennent une large place (ibid. : IX). L'auteur rappelle cependant son souci de les replacer «dans leur contexte culturel d'origine et d'examiner leurs connexions multiples avec la formation des idées dans l'ancienne philosophie indienne, la cosmologie, la religion, la littérature, la science et tous les autres domaines signifiants de la pensée " (ibid.). Ces multiples liens permettent à l'auteur de reconstituer ce qu'il appelle une "pensée musicale " (ibid. : 6), c'est-à-dire «la structure globale des idées environnant et informant la pratique de la musique" (ibid.). Dans ce cadre, Lewis E. Rowell traite du sens accordé à la nature du son, des valeurs attachées à la transmission, de l'influence des doctrines tantriques et du shivaïsme du Kashmir.

Cependant, la notion même de "pratique musicale » demeure obscure lorsque les musiques étudiées ne sont plus vivantes. Les auteurs sont alors conduits à spéculer sur des liens éventuels avec la pratique actuelle des musiques classiques. Selon Lewis E. Rowell, s'il s'agit d'extraire «les racines originelles des concepts musicaux et des pratiques modernes indiennes, l'attention principale doit toujours se porter sur ce qu'était la musique indienne [...] et non sur ce qu'elle est " (ibid. : 5) ${ }^{2}$. Pourtant, force est de constater qu'une notation musicale ne saurait à elle seule renseigner sur les dimensions sensibles, acoustiques et orales d'un énoncé musical. "Pour prétendre connaître ce à quoi pouvait ressembler la musique de l'Inde ancienne, la connaissance doit s'appuyer sur deux sources: les données textuelles et la musique indienne telle qu'on peut l'entendre aujourd'hui " (ibid.: 7). Là se situe sans aucun doute la richesse singulière du terrain classique indien mais aussi, on l'aura compris, son principal écueil sur le plan méthodologique.

Le terme d' "ethnomusicologie historique " a été définitivement adopté par Bonnie C. Wade (1998: LVI) dans un ouvrage consacré aux sources iconographiques de la période moghole. L'auteur propose d'ouvrir un nouveau champ d'étude après s'être consacrée quelques années plus tôt à certains aspects de la

2. Italiques de l'auteur. 
performance contemporaine du genre vocal classique khyàl (1984). Lauteur reconstitue la vie musicale à la cour moghole, telle que nous la décrivaient les peintres patronnés par l'empereur Akbar. Portraits, miniatures et récits littéraires informent sur l'usage des instruments de musique, la composition des orchestres, les audiences et certains aspects de la performance au travers de scènes conventionnelles comme les batailles, les mariages, les processions et les banquets. Loin de se limiter aux seules méthodes de l'histoire de l'art, Bonnie C. Wade s'interroge aussi sur l'ensemble du contexte politique et culturel de l'époque dans une perspective proprement "ethnomusicologique" (ibid. : LIV). Ainsi apprend-on que la production artistique patronnée par les différents empereurs constitue un lieu central d'expression et d'assise de leur pouvoir politique. L'auteur explore également la question de la place des femmes du harem dans la production musicale, et en dernier lieu, restitue le processus d'indianisation progressive de la culture musicale moghole au cours des siècles. Bonnie C. Wade montre ainsi que «les sources écrites, à la fois émiques et étiques [...] contribuent à définir les paramètres historiques et les contextes culturels pour une compréhension de l'Empire moghol et de ses dirigeants » (ibid.: L). Le " texte " devient par là même un véritable terrain anthropologique. Un jalon est posé pour l'ethnomusicologie des musiques du passé, sans perdre de vue cependant qu'elles ont contribué à l'émergence d'une culture musicale nord-indienne aujourd'hui bien vivante.

La musicologie historique réintègre sans aucun doute dans l'analyse le volet " culturel » du modèle de pensée merriamiste mais l'approche se heurte par définition à l'incomplétude des données proprement musicales. Quant aux nombreuses recherches portant sur les pratiques actuelles de la musique classique, elles traitent presque exclusivement du système musical, autrement dit de la grammaire interne à la musique (music itself).

\section{La musique "à côté" de la culture? L'analyse des traditions classiques contemporaines}

En tant que musiques d'"art», les musiques classiques encore appelées "musiques de traité" (śastrìya sanigìta), constituent le champ le plus abondamment exploré dans les dernières décennies. Enseignées de nos jours dans les universités et les académies, les musiques classiques conferent bien souvent à ceux qui les pratiquent le statut de musicologue, statut intimement lié à celui de musicien professionnel. La publication de R. C. Mehta (1994) issue d'un séminaire de réflexion sur les perspectives et les projets futurs de la recherche musicale, est assez révélatrice sur ce point. Les universitaires et artistes indiens ayant contribué au numéro partagent, semble-t-il, un même type de discours sur la musique du fait de leur double compétence à la fois musicale (pratique vocale ou instrumentale) et théorique. Les Indiens nous démontrent que seule la pratique d'une musique nous habilite à en analyser le système musical, l'esthétique, les techniques de jeu et même les concepts d'innovation et de tradition. Voilà un cas singulier de musiques où la réflexivité des musiciens est érigée en tant que science 
musicologique à part entière. La configuration indienne de la recherche dévoile cependant ses limites lorsqu'elle analyse, à travers ses catégories, des répertoires autres que les siens, comme par exemple les musiques populaires (Guillebaud 2003) ou celles des populations ādivāsī. En Inde, les deux grands systèmes hindoustani et carnatique forment le seul et unique soubassement des musicologies académiques dominantes.

Dans une tradition où théorie et pratique relèvent, pour ainsi dire, d'une même sphère de pensée, il n'est guère étonnant que les spécialistes occidentaux actuels des musiques classiques indiennes soient aussi et d'abord des musiciens. Leur entrée dans l'univers esthétique de ces musiques correspond souvent à celle du disciple engagé dans une relation de transmission intime et de longue durée avec un maître. C'est le cas par exemple de Patrick Moutal qui propose une étude générale des «mécanismes de base » de la musique hindoustanie (1987). Celle-ci a été complétée quelques années plus tard par une approche comparative de cinq familles de ràga (1991), accompagnée d'un index (1991), dans un format plus proche de la compilation, où chaque ràga est présenté par son échelle, ses degrés "parlants" (vàdi) et son ornementation. L'analyse du matériel mélodique se fonde exclusivement sur la pratique actuelle des ràga hindoustanis, accessibles par le biais de très nombreux enregistrements et par les analyses publiées par ses collègues indiens en langue hindi.

De nombreux autres musiciens-auteurs (Bor 1999 ; Clayton 2000 ; Slawek 1987), héritiers d'une longue tradition de recherche marquée par des personnalités indiennes comme Vishnu N. Bhatkande, Subbha. B. Rao, Pichu. Sambamurthy ou Nazir A. Jairazbhoy, ont contribué à inclure la musique dite "de traité» dans l'étude des musiques «en performance", cette fameuse dimension manquant aux (ethno)-musicologues historiens. Au regard de l'approche merriamiste, ces études présentent une certaine incomplétude, car la musique ne se réfere qu'à elle-même. Disjoint du sonore, le contexte est lui aussi autoréférencé. La dichotomie entre ce qui relèverait de l'interne et de l'externe à la musique, reprend ici furtivement sa place.

J'ai évoqué plus haut l'abondance des travaux portant spécifiquement sur le musical. Plus rares sont ceux qui font prévaloir l'analyse culturelle (Neuman 1990 ; Kippen 1988). Très novateur, l'ouvrage de Daniel M. Neuman décrit la "structure culturelle" de la tradition hindoustanie, encore appelée "culture musicale ", qu'il définit comme un "système de symboles et de significations que les musiciens utilisent et exposent en référence à leur musique et leur identité de musicien " (1990 : 24). Ce système comprend notamment le sens de la pratique, la relation affective au maître et le rôle du divin. Ces éléments de discours appartiennent à l'identité du musicien et sont impliqués dans la création musicale. L'auteur s'attache ensuite à décrire l'organisation sociale des musiciens en "écoles" (gharànà) à partir de matériaux généalogiques. Daniel M. Neuman souligne l'importance de leur "identité socio-musicale», définie comme "une identité sociale basée sur un héritage musical» (ibid.: 44). Enfin, un essai d' "écologie de la musique hindoustani " présente en fin d'ouvrage les différentes 
stratégies adaptatives des musiciens aux nouveaux réseaux de patronage, comme par exemple ceux de la radio gouvernementale (All India Radio).

\section{Les genres "classiques" versus les genres "non classiques": étanchéité des classifications ou vecteurs d'une même indianité?}

Le versant "non classique " des musiques du sous-continent, relevant traditionnellement des études folkloriques, semble réhabilité aujourd'hui comme objet ethnomusicologique à part entière (Capwell 1986; Henry 1988; Prasad 1985 ; Tingey 1994). Des réflexions récentes ayant trait à la catégorisation des musiques "populaires " et "tribales ", avec toutes les précautions que nécessite l'emploi de tels termes, réintègrent l'analyse de la musique dans son contexte culturel de production.

Carol Babiracki (1991) a récemment souligné la dissymétrie flagrante existant dans la recherche indianiste entre les études portant sur les genres classiques et celles se consacrant aux musiques folk et tribal. L'auteur interprète ce déséquilibre, à la fois quantitatif et qualitatif, à la lumière du grand partage opéré dans l'indianisme, au cours des années 1950, entre "Grande " et "Petite » traditions. La documentation portant sur les musiques de village a commencé au XVIII siècle avec le travail des folkloristes. Les textes de chants ont été collectés et publiés sous forme de recueils sans aucune mention du contexte de leur exécution. À partir du XIX ${ }^{e}$ siècle, les compilations ethnographiques fournissent d'amples descriptions des instruments de musique, des rituels et des festivals sans réellement mettre en perspective ces différents éléments. Dans ces deux types d'approche, l'accent a été mis sur les "textes et les contextes des expressions musicales plus que sur leur structure musicale» (ibid. : 69). L'objectif de Carol M. Babiracki n'est pas tant de rediscuter les notions de Grande et Petite traditions - partage qu'elle semble implicitement défendre d'un point de vue méthodologique -, mais d'examiner s' "il y a sens à parler de "musique tribale" comme une catégorie de musique indienne distincte des autres traditions de village" (ibid. : 72). L'auteur répond avec précaution, que les «musiques tribales et les musiques folk non tribales different dans la relation quelles entretiennent chacune avec la Grande tradition, les musiques classiques " (ibid. : 76). La démarche est novatrice car elle considère le sonore comme critère premier dans la classification par genre. Cependant, dans son projet d'isoler le genre "tribal ", Carol M. Babiracki succombe à son insu au grand partage entre Grande et Petite traditions.

Cette question a été rediscutée collectivement dans un numéro spécial de la revue Asian Music consacré à la "Musique tribale de l'Inde" (Babiracki et al. 2001) et réunissant les ethnomusicologues Carol M. Babiracki, Roderic Knight, David Roche et Richard Wolf, tous spécialistes de différentes populations ādivāsī (Kota, Munda, Mina et Pardhan-Gond). Il s'agit là encore d'évaluer la validité de l'expression « musique tribale », utilisée dans l'article de Roderic Knight (ibid. : 2). L'article de Wolf inventorie trois perspectives ethnomusicologiques possibles pour étudier la notion même de "tribu " (ibid. : 5). La première consisterait à travailler 
spécifiquement sur la façon dont on se représente la tribu dans le discours institutionnel ou dans les médias (popular-representational approach). Une deuxième approche "on the ground» (ibid. : 12) consisterait à confronter les "concepts et les catégories employées par des personnes vivant dans un cadre multi-ethnique" (ibid. : 5). La troisième approche dite "synthétique " (ibid. : 17) étudie la musique elle-même dans l'objectif de révéler "dans quelle mesure en Inde les différentes traditions qu'on appelle "tribales", partagent des éléments de style ou de structure, et d'évaluer l'importance de ces éléments communs" (ibid. : 5).

Onkar Prasad, auteur d'une étude sur la musique des Santal (1985), fait partie aussi de ces rares chercheurs abordant les répertoires des populations ādivāsī par le biais du son lui-même. À la différence de ses collègues occidentaux écrivant sur portée, Onkar Prasad travaille en notation indienne et applique un modèle d'analyse formalisé précédemment pour l'étude de ràga hindoustani (système Sanyal). Dans un autre ouvrage consacré au paysage musical de Bénarès, Onkar Prasad (1987) discute aussi largement des classifications par genre. Il établit un premier niveau d'opposition recouvrant en partie le partage entre Grande et Petite traditions (ibid. : 1) : dans la catégorie "local, indigène, du pays » (dêsí), les musiques folk (ou laukic) et tribal se différencient de la catégorie màrga où sont classées les traditions classiques (ou shastric) À un second niveau, de nouvelles catégories de classement émergent comme les musiques semi-classiques (upashastric) et les musiques de film (ibid.). Bien que le vocabulaire qu'il emploie ne soit pas toujours des plus heureux et malgré sa volonté affirmée de dépasser une certaine vision évolutionniste des classifications (ibid.: 10), Onkar Prasad entend mettre le doigt sur la "continuité et la persistance de la civilisation indienne » et insiste sur la nécessité de les comprendre "au-delà de la dichotomie entre folk et urbain, folk et classique, et pureté et pollution " (ibid. : 6). Le projet vise implicitement la mise en valeur d'une certaine unité culturelle indienne par le biais de classifications qui servent de vecteurs idéologiques. Les termes d' "universalisation » et de "particularisation " (parochialisation) employés par Onkar Prasad révèlent toute la difficulté à trouver un compromis dynamique pour rendre compte en même temps de la diversité des musiques existantes et de leur perméabilité.

Le thème reste sensible et resurgit dans de nombreux autres travaux sur la musique. Prem Lata Sharma (1994) affirme par exemple que «la division des populations en tribale, rurale et urbaine et l'étiquetage des musiques en tribale, folk et classique est le produit de l'influence de la pensée occidentale. La pensée traditionnelle indienne n'a jamais analysé le musical, ni aucune autre activité, dans une vision d'ordre hiérarchique " (ibid.: 4). L'idéologie sousjacente dépasse certainement le seul domaine des études musicales mais révèle aussi, à mon sens, la tâche urgente qui incombe aux ethnomusicologues indianistes de dépasser les problèmes de classification. De même, nombreux sont les articles traitant des différents genres, notamment en terme d'interpénétration (Allen 1998; Groesbeck 1999; Thompson 1995). 
D'autres auteurs, ayant pris leurs distances par rapport aux débats de catégorisation, proposent des modèles d'analyse de la performance, où la musique et son contexte sont appréhendés en terme de processus d'actualisation mutuelle.

\section{Le performatisme en ethnomusicologie: la musique "en dehors" d'elle-même}

L'ouvrage de Regula Qureshi consacré au qawwali (1986) inaugure un nouveau modèle d'analyse globale des faits musicaux en performance. Dans une assemblée de qawwali, les interactions entre les musiciens et les auditeurs imposent une structure fluide et mouvante à la musique. La forme d'un chant, par exemple, varie d'une performance à l'autre en fonction de l'activité de l'auditoire en expérience d'extase: le musicien est amené à répéter, adjoindre ou omettre certaines unités musicales pour satisfaire au mieux ses auditeurs. La structure même de la musique est constamment réactualisée à travers différentes interactions. Il s'agit bien pour Regula Qureshi de traiter spécifiquement du lien entre la musique et son contexte de production, c'est à dire " comment la dimension contextuelle est indispensable à la compréhension de la musique et comment précisément ce sont les significations extra-musicales inhérentes au sonore qui donnent à la musique son pouvoir d'affecter à son tour le contexte » (Qureshi 1986: XIV). Pour y répondre, l'auteur propose un modèle d'analyse en trois temps. La première étape, consiste à dégager la structure musicale du qawwali (ibid. : 11), autrement dit " un système de règles autonome pour générer la musique en performance" (ibid.: 6). La formule est novatrice car elle souligne l'aspect génératif de la grammaire musicale plutôt que son caractère interne par rapport à un contexte qui lui serait externe.

La deuxième étape vise à identifier le contexte de la performance, la situation globale dans laquelle la musique est produite et les dynamiques sociales et culturelles qui y sont impliquées (ibid.). Le qawwali est ici décrit comme un rituel religieux dont la structure et les normes relèvent de l'idéologie soufie. Celle-ci se caractérise par une conception particulière du divin, une hiérarchie des saints, une forme d'expression poétique et une certaine organisation socioéconomique de ses institutions. Ces éléments culturels donnent sens au contexte immédiat de la performance: sa structuration en séquences, son assemblée hiérarchisée, la nature mystique de l'expérience d'écoute ainsi que les règles entourant les offrandes d'argent aux musiciens.

Enfin, la troisième étape traite du "processus de performance " (ibid.: 11) au cours duquel les éléments contextuels agissent sur la musique elle-même. Lauteur montre comment le musicien sélectionne les différentes variables musicales (intensification, répétition, adjonction...) de manière stratégique afin de répondre aux attentes des auditeurs tout en s'assurant un maximum de revenus financiers (ibid. : 140). L’analyse, basée sur un vaste matériel vidéo, est 
remarquable tant par la finesse de l'observation des différentes interactions entre musiciens et auditeurs, que par sa présentation graphique ${ }^{3}$.

Regula Qureshi montre ainsi que la musique et son contexte peuvent être analysés "en compatibilité l'un avec l'autre" (ibid. : 9) sans qu'il soit nécessaire à aucun moment d'opérer une dichotomie entre l'approche musicologique et anthropologique. En établissant un lien processuel et interactif entre la musique et son contexte, l'auteur rend obsolète la distinction entre ce qui relèverait de l'interne et de l'externe. Dans ce processus, le musicien occupe une place centrale, il "utilise la musique pour exprimer le contexte et le manipuler" (ibid. : 207).

En 1991, Pirkko Moisala propose en dernière partie de son ouvrage consacré à la culture musicale Gurung (Népal central) un autre modèle performatif d'analyse de la musique. Nourrie des travaux de Marcia Herndon, l'auteur suggère de voir l'occasion musicale (ou performance) comme "une incorporation (encapsulation) de la cognition culturelle» (Moisala 1991 : 380). L'originalité de l'étude tient surtout à son approche diachronique des matériaux collectés entre 1975 et 1985 dans le même village. Elle permet à l'auteur de traiter des changements musicaux survenus à cette période et imputables aux contraintes d'intégration nationale de la population gurung à l'État népali. Comme l'explique Pirkko Moisala, ces changements sont "concomitants avec ceux des prédispositions cognitives (cognitive premises) de la culture» (ibid. : 373). En effet, la situation culturelle conflictuelle dans laquelle vivent les Gurung se reflète au sein même de leurs activités musicales, qui incluent à la fois les répertoires anciens et les musiques pan-népalaises adoptées des mass media. L'auteur y voit l'imbrication de deux sphères cognitives, celles de l'"ancien" et du "nouveau ", catégories employées par ailleurs par les Gurung eux-mêmes (ibid. : 358).

Le modèle de Pirkko Moisala intègre l'approche cognitive à l'étude de la performance. À ce titre, il injecte du culturel dans l'étude de la cognition musicale, domaine jusqu'ici investi par la psychologie. Il s'agit en effet de dépasser l'analyse des seuls processus mentaux de la perception musicale pour s'intéresser plus largement aux processus cognitifs déployés par le musicien au moment même de la performance (ibid. : 383). La notion de contexte est elle aussi redéfinie. Comme l'explique l'auteur, cette notion doit être envisagée sur trois niveaux différents que sont la culture, la tradition musicale et l'occasion musicale proprement dite. Pirkko Moisala y fait correspondre trois domaines de connaissance particulière que possède le musicien : esthétique, croyances et normes (niveau de la culture); matériel rythmique, mélodique et kinésique (niveau de la tradition); et enfin technique et style (niveau de l'occasion). Non actualisées au moment de la performance ("processus non temporel»), ces connaissances relèvent de la mémoire « inactive " ou à long terme du musicien. Parallèlement, le musicien opère au moment même où il joue des regroupements rythmiques, des combinaisons et

3. Des videographs montrent sur la même échelle temporelle la transcription sur portée de la mélodie chantée et les gestes ou actions des différents participants; des videocharts détaillent l'impact que produit chaque petit réarrangement musical sur les auditeurs, de même que l'influence que peuvent avoir à leur tour les gestes du public sur la forme musicale. 
synthèses mélodiques, des chaînes d'unité, etc. Ces compétences actualisées au moment même de la situation de jeu ("processus temporel ») sont stockées dans une mémoire à court terme appelée "active». Par cette modélisation, Pirkko Moisala propose une étude globale des procédés mentaux universaux en lien avec les données contextuelles. L'articulation qui unit la musique et son contexte est pensée ici comme la rencontre in situ de différents éléments compartimentés de notre mémoire.

Chez Regula Qureshi et Pirkko Moisala, la performance est l'unité d'observation privilégiée. D’autres auteurs, spécialistes des musiques dites "populaires» (popular musics), proposent de ré-interroger cette notion à la lumière des musiques produites et diffusées par les mass media.

\section{Ethnomusicologie des popular musics : nouveaux enjeux}

Les musiques médiatisées par l'industrie du film ou de la cassette occupent une place de plus en plus croissante dans le paysage musical du sous-continent. Évoquées souvent dans les débats de classification par genre, elles font aujourd'hui l'objet de recherches spécifiques (Arnold 1993; Grandin 1989; Manuel 1993). Ce n'est plus tant la nature du lien qui s'articule entre musique et société qui est traité mais diverses thématiques parfois proches de la sociologie musicale. Comme l'explique Peter L. Manuel dans son ouvrage consacré à l'industrie de la cassette dans le Nord de l'Inde, le paradigme merriamiste "était orienté vers l'étude de la musique dans des sociétés isolées et sans classes " et peut paraître "problématique quand sont concernées les sociétés complexes [...] Le défaut d'un paradigme supposant l'uniformité sociale et la cohésion devient plus évident encore quand sont concernés les mass media - comme ils le sont actuellement dans la plupart des sociétés» (Manuel 1993: 5). L’ouvrage explore les changements qui se sont opérés dans les popular musics à la suite du développement de l'industrie de la cassette dans les années 1970. Le passage d'un marché unique monopolisé par la Gramophone Company of India à un réseau multipolaire de petits producteurs locaux, a eu un impact fort sur le ghazal moderne, la musique dévotionnelle, les musiques régionales et le genre folk rasiya. Peter L. Manuel tisse un vaste panorama des réseaux de production, de commercialisation et de consommation des musiques enregistrées et pose, pour la première fois, la question de l'esthétique de ces musiques et de leur lien avec certains mouvements sociopolitiques et les conflits communalistes. Un chapitre important sur les "politiques de la parodie" (ibid.: 131-152) traite des processus musicaux d'emprunts mélodiques, des parodies commercialisées sur cassette et des questions de copyright. La notion d' " emprunt» mériterait sans doute d'être exploitée aussi par les chercheurs traitant, on l'a vu, des classifications par genre.

Ingmar Grandin (1991), dans un ouvrage consacré à la vie musicale dans un quartier newar de la ville de Kirtipur (Népal), propose de redéfinir la notion de "pratique musicale " comme l'ensemble des "actions " liées à la musique, c'està-dire la performance, mais aussi le fait de l'écouter (Grandin 1991 : XII). Cette 
pratique comprend au sens large "toutes les activités qui impliquent des ressources musicales" (ibid.) comme par exemple écouter la radio, acheter des cassettes ou se rendre à une manifestation musicale. En considérant l'ensemble de ces ressources utilisées à l'échelle d'un quartier, l'auteur montre que l'étude de la musique en performance n'apporte qu'un point de vue très limité par rapport à la réalité des diverses occasions musicales. Dans cet ouvrage, le terme "performance " s'applique seulement à la dimension du savoir-faire musical alors que le terme de "pratique ", inclut plus largement les situations d'écoute relevant du même domaine d'action musicale. Sans réellement se focaliser sur un auditoire d'une performance musicale donnée - ce qui équivaudrait finalement à morceler l'unité d'observation qu'ont choisie d'autres ethnomusicologues (Qureshi 1986 ; Moisala 1991) - Ingemar Grandin propose de mettre en perspective l'ensemble des "occasions musicales" se tenant dans un même quartier newar et impliquant à chaque fois des ensembles instrumentaux différents, des répertoires et des situations d'écoute distincts. L'occasion est définie de manière large comme toute "situation sociale où la musique est présente " (ibid. : XV), les éléments musicaux étant qualifiés d' "événements" parmi d'autres au sein de la situation (ibid.). Ingemar Grandin décrit donc l'ensemble de ces occasions durant lesquelles les gens d'un même quartier font et entendent de la musique. Parmi celles-ci, l'auteur distingue les «musiques de performance quotidiennes" (hymnes du matin, veillées...) des «situations quotidiennes de non performance " comme par exemple écouter Radio Nepal, des cassettes ou regarder un film (ibid. : 1-28). À côté des situations quotidiennes, les « occasions extra-ordinaires" (processions rituelles ou de mariage, élection politique, festival de musique sur scène) ont la caractéristique d'inclure à la fois des situations de performance et d'écoute (ibid. : 28-61).

Ingemar Grandin fonde sa connaissance des musiques locales newar sur les travaux antérieurs menés par d'autres chercheurs et mène son observation de la vie musicale du quartier selon les méthodes de la sociologie quantitative ${ }^{4}$. La procédure d'enquête est assez lointaine des méthodes de l'observation ethnographique, ce qui appauvrit sans doute la réflexion finale sur la question de la continuité et du changement. Plus intéressante est l'analyse du processus de «medialization» défini comme «l'assimilation et l'adaptation de nouvelles ressources - les médias eux-mêmes et leurs contenus [...] - dans la pratique musicale et socioculturelle " (ibid. : XIII, 184), c'est-à-dire dans « un modèle existant » (ibid.). L'ouvrage a par ailleurs le mérite d'interroger les musiques médiatisées et les répertoires modernes joués et/ou entendus par les Newar dans une même et unique sphère de pratique, où l'ancien et le nouveau participent de choix identitaires complexes.

4. Des figures et des tableaux montrent par exemple la composition par classe d'âge de la population du quartier ayant appris les chants dévotionnels bhajan (Grandin 1991 : 100) ; des statistiques détaillent le nombre de propriétaires de radio et/ou de magnétophone et le temps passé à l'écouter (ibid. : 138-139) ; une table des goût musicaux et des motivations est établie à partir de questionnaires directifs (ibid. : 171-174). 
La musicologie historique et l'étude des traditions classiques contemporaines apparaissent souvent comme complémentaires dans leurs réflexions sur la dialectique entre théorie et pratique. Si la première semble s'intéresser plus volontiers aujourd'hui au contexte culturel des sources musicologiques qu'elle étudie, la seconde focalise son attention tantôt sur la musique elle-même tantôt sur son contexte de production, mais très rarement simultanément sur ces deux dimensions. Longtemps restées minoritaires, les études portant sur les musiques non classiques ont tendance à construire leurs modèles en fonction des caractéristiques formelles des musiques qu'elles prennent pour objet (folk, tribal), plutôt que par rapport à un paradigme d'analyse du phénomène musical. Il n'en reste pas moins que le paysage musical du sous-continent, en raison de sa complexité, a généré des réflexions conceptuelles novatrices, notamment à partir des popular musics, ainsi que des propositions d'analyse globale de la musique, devenues des repères incontournables pour la discipline.

MOTS CLÉS/KEYWORDS : musique/music - histoire/history - caste - tribus/tribes - performance - médias.

\section{BIBLIOGRAPHIE}

Allen, Matthew

1998 "Tales Tunes Tell : Deepening the Dialogue between "Classical" and "NonClassical" in the Music of India ", Yearbook of Traditional Music 30 : 22-52.

Arnold, Alison E.

1993 "Aspects of Production and Consumption in the Popular Hindi Film Song Industry ", Asian Music 24 (1) : 122-136.

Arnold, Alison E., ed.

2000 The Garland Encyclopedia of World Music. V: South Asia. The Indian Subcontinent. New York, Garland [avec $1 \mathrm{CD}$ ].

Babiracki, Carol M.

1991 "Tribal Music in the Study of Great and Little Traditions of Indian Music ", in Bruno Nettl \& Philip V. Bohlman, eds, Comparative Musicology and Anthropology of
Music: Essays on the History

of Ethnomusicology. Chicago,

The University of Chicago Press : 69-90.

Babiracki, Carol M. et al., eds

2001 "Tribal Music of India ", Asian Music 32 (1).

Bernède, Franck, ed.

1997 The European Bulletin of Himalayan Research 12-13: Himalayan Music,

State of the Art.

Bor, Joep

1986-1987 "The Voice of the Sarangi :

An Illustrated History of Bowing in India ", National Center for the Performing Arts, Quarterly Journal 15 (3-4) et 16 (1).

1999 The Raga Guide: A Survey of 74 Hindustani Ragas. Charlottesville, Nimbus Records [avec 4 CD]. 


\section{Capwell, Charles}

1986 The Music of the Bauls of Bengal.

Ohio, The Kent State University Press.

Clayton, Martin

2000 Time in Indian Music: Rhythm,

Metre, and Form in North Indian Rag

Performance. New York, Oxford University

Press [avec $1 \mathrm{CD}]$.

\section{Farrell, Gerry}

1999 [1997] Indian Music and the West.

Oxford, Oxford University Press.

Grandin, Ingemar

1989 Music and Media in Local Life. Music Practice in a Newar Neighbourhood in Nepal, Linköping (Suède), Linköping University, ( Linköping Studies in Arts

and Science $41 »)$.

\section{Groesbeck, Rolf}

1999 "Classical Music", "Folk Music", and the Brahmanical Temple in Kerala, India ", Asian Music 30 (2) : 87-112.

\section{Guillebaud, Christine}

2003 «Des théories musicales et de leurs croisements en Inde du Sud", in JeanJacques Nattiez, ed., Enciclopedia della musica, III : Musiques et cultures. Turin, Einaudi : 616-642.

\section{Henry, Edward O.}

1988 Chant the Names of God: Musical Culture in Bhojpuri-Speaking India. San Diego, San Diego State University Press.

Jairazbhoy, Nazir A.

1993 "South Asia. India » in Helen Myers, ed., Ethnomusicology: Historical and Regional Studies, The New Grove Handbooks in Music. New-York-London, Norton : 274293.

Kippen, James R.

1988 The Tabla of Lucknow: A cultural Analysis of a Musical Tradition. Cambridge, Cambridge University Press.
Manuel, Peter L.

1989 Thumri in Historical and Stylistic Perspectives. Delhi, Motilal Banarasidass.

1993 Cassette Culture: Popular Music and Technology in North India. Chicago, University of Chicago Press.

Mehta, Ramanlal C. ed.

1994 Music Research. Perspectives and Prospects-Reference Indian Music. Bombay Baroda, Indian Musicological Society.

Moisala, Pirkko

1991 Cultural Cognition in Music. Continuity and Change in the Gurung Music of Nepal. Jyväskylä, Gummerus Kirjapaino Oy ("Suomen etnomusikologisen seuran julkaisuja » 4).

Moutal, Patrick

1987 Hindustānī Rāga Sañgìta. Une étude de quelques mécanismes de base. Paris,

CEMO.

1991 A Comparative Study of Selected Hindustānī Rāga Sañgita Based on Contemporary Practice. New-Delhi, Munshiram Manoharlal Publishers.

Neuman, Daniel M.

1990 [1980] The Life of Music in North India. The Organization of an Artistic Tradition. Chicago, The University of Chicago Press.

\section{Prasad, Onkar}

1985 Santal Music: A Study in Pattern and Process of Cultural Persistence. New Delhi, Inter-India Publications.

1987 Folk Music and Folk Dances of Banaras. Calcutta, Anthropological Survey of India.

Qureshi, Regula B.

1986 Sufi Music of India and Pakistan: Sound, Context and Meaning in Qawwali. New York, Cambridge University Press [avec 1 cassette audio, rééd. 1995 avec CD]. 
1987 «Musical Sound and Contextual Input: A Performance Model for Musical Analysis ", Ethnomusicology 31(1) : 56-86.

1991 «Whose Music? Sources and Contexts in Indic Musicology", in Bruno Nettl \& Philip V. Bohlman, eds, Comparative Musicology and Anthropology of Music: Essays on the History of Ethnomusicology. Chicago,

The University of Chicago Press : 152-168.

Ranade, Ashok D.

2000 Journal of the Indian Musicological Society 31 : Reflections on Musicology and History-with reference to Hindustani Music.

\section{Rowell Lewis Eugene}

1992 Music and Musical Thought in Early India. Chicago, University of Chicago Press, ("Chicago Studies in Ethnomusicology»).

\section{Sharma, Prem Lata}

1994 "An Agend For Music Research: Some Thrust Areas » in R. C. Metha, ed., Music Research. Perspectives and ProspectsReference Indian Music. Bombay Baroda, Indian Musicological Societ :1-9.

\section{Slaweck, Stephen M.}

1987 Sitàr Technique in Nibaddh Forms. New-Delhi, Motilal Banarsidass.

Thompson, Gordon R.

1995 "What's in a Dhal ? Evidence of Ragalike Approaches in a Gujarati Musical Tradition », Ethnomusicology 39 (3) : 417-432.

\section{Tingey, Carol}

1994 Auspicious Music in a Changing

Society: The Damài Musicians of Nepal.

Series $2 »)$.

\section{Wade, Bonnie C.}

1984 Khyàl: Creativity within North India's

Classical Music Tradition. Cambrige,

Cambridge University Press, ("Cambridge Studies in Ethnomusicology ") [avec 1 cassette audio].

1998 Imaging Sound: An Ethnomusicological Study of Music, Art and Culture in Mughal India. Chicago, University of Chicago Press,

("Chicago Studies in Ethnomusicology»).

\section{Wayne, Howard}

1986 Veda Recitation in Vārānāṣ̄i. Delhi, Motilal Banarsidass.

\section{Weisethaunet, Hans}

1998 The Performance of Everyday Life: The Gàine of Nepal. Oslo, Scandinavian University Press.

\section{Widdess, Richard}

1995 The Ràgas of Early Indian Music. Modes, Melodies and Musical Notations from the Gupta Period to c. 1250. New-York, Oxford University PressClarendon Press. 